University of Wollongong

Research Online

Australian Institute for Innovative Materials -

Papers

Australian Institute for Innovative Materials

2001

\title{
Distributed polarizability of the water dimer: Field-induced charge transfer along the hydrogen bond
}

Marc in het Panhuis

University of Wollongong, panhuis@uow.edu.au

P L A Popelier

UMIST

R W. Munn

UMIST

J G. Angyan

Universite Henri Poincare (Nancy I)

Follow this and additional works at: https://ro.uow.edu.au/aiimpapers

Part of the Engineering Commons, and the Physical Sciences and Mathematics Commons

Research Online is the open access institutional repository for the University of Wollongong. For further information contact the UOW Library: research-pubs@uow.edu.au 


\title{
Distributed polarizability of the water dimer: Field-induced charge transfer along the hydrogen bond
}

\author{
Abstract \\ The topological partitioning of electronic properties approach at Hartree-Fock level is used to investigate \\ charge transfer response in a water dimer. Distributed polarizability components are employed to \\ calculate the change in electron density under external fields. Field-induced charge flow between the \\ water monomers is most significant along the direction of the hydrogen bond. The molecular \\ polarizability of the molecules in the dimer is reduced owing to formation of the hydrogen bond.

\section{Keywords} \\ polarizability, transfer, distributed, along, hydrogen, bond, induced, field, dimer, water, charge \\ Disciplines \\ Engineering | Physical Sciences and Mathematics

\section{Publication Details} \\ in het Panhuis, M., Popelier, P. L. A., Munn, R. W. \& Angyan, J. G. (2001). Distributed polarizability of the \\ water dimer: Field-induced charge transfer along the hydrogen bond. Journal of Chemical Physics, 114 \\ (18), 7951-7961.
}




\section{A|P|l $\begin{aligned} & \text { The Journal of } \\ & \text { Chemical Physics }\end{aligned}$}

\section{Distributed polarizability of the water dimer: Field-induced charge transfer along the hydrogen bond}

M. in het Panhuis, P. L. A. Popelier, R. W. Munn, and J. G. Ángyán

Citation: The Journal of Chemical Physics 114, 7951 (2001); doi: 10.1063/1.1361247

View online: $\mathrm{http}: / / \mathrm{dx}$.doi.org/10.1063/1.1361247

View Table of Contents: http://scitation.aip.org/content/aip/journal/jcp/114/18?ver=pdfcov

Published by the AIP Publishing

\section{Articles you may be interested in}

Effect of an external electric field on the dissociation energy and the electron density properties: The case of the hydrogen bonded dimer HF HF a)

J. Chem. Phys. 130, 044104 (2009); 10.1063/1.3065972

Hydrogen bonds in liquid water studied by photoelectron spectroscopy

J. Chem. Phys. 126, 124504 (2007); 10.1063/1.2710792

Hydrogen bonding and induced dipole moments in water: Predictions from the Gaussian charge polarizable model and Car-Parrinello molecular dynamics

J. Chem. Phys. 125, 144519 (2006); 10.1063/1.2355485

Development of transferable interaction models for water. IV. A flexible, all-atom polarizable potential (TTM2-F) based on geometry dependent charges derived from an ab initio monomer dipole moment surface

J. Chem. Phys. 116, 5115 (2002); 10.1063/1.1447904

Determination of vibrational polarizabilities and hyperpolarizabilities using field-induced coordinates

J. Chem. Phys. 113, 5203 (2000); 10.1063/1.1290022

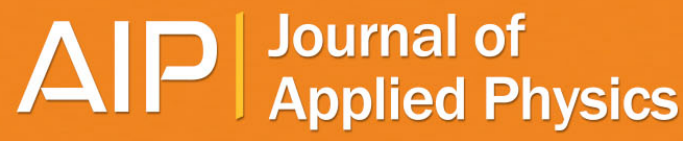

Journal of Applied Physics is pleased to announce André Anders as its new Editor-in-Chief 


\title{
Distributed polarizability of the water dimer: Field-induced charge transfer along the hydrogen bond
}

\author{
M. in het Panhuis, ${ }^{\text {a) }}$ P. L. A. Popelier, ${ }^{\text {b) }}$ and R. W. Munn ${ }^{\text {c) }}$ \\ Department of Chemistry, UMIST, Manchester M60 1QD, United Kingdom \\ J. G. Ángyán d) \\ Laboratoire de Chimie Théorique, Université Henri Poincaré, B.P. 239, \\ 54506 Vandauvre-lès-Nancy, France
}

(Received 1 November 2000; accepted 15 February 2001)

\begin{abstract}
The topological partitioning of electronic properties approach at Hartree-Fock level is used to investigate charge transfer response in a water dimer. Distributed polarizability components are employed to calculate the change in electron density under external fields. Field-induced charge flow between the water monomers is most significant along the direction of the hydrogen bond. The molecular polarizability of the molecules in the dimer is reduced owing to formation of the hydrogen bond. (C) 2001 American Institute of Physics. [DOI: 10.1063/1.1361247]
\end{abstract}

\section{INTRODUCTION}

The polarizability of a molecular system describes the response of the system to electric fields. It is important for understanding various phenomena, including intermolecular forces and optical and dielectric properties. One aspect of the polarizability that has attracted increasing attention in recent years has been its distribution over the molecule. This has two aspects. One is the practical aspect that the phenomena where polarizability is important, for example intermolecular forces between adjacent molecules, involve electric fields that vary significantly over molecular length scales and hence cannot be characterized by a simple average polarizability. The other, conceptual, aspect is that the polarizability distribution contains information of chemical significance about how the constituent atoms contribute to the overall response.

A general theory of distributed polarizability based on distributed multipole analysis was developed by Stone. ${ }^{1}$ Subsequently Ángyán et al., ${ }^{2}$ using Bader's theory of Atoms in Molecules (AIM) $)^{3,4}$ and the coupled perturbed Hartree-Fock (CPHF) approximation ${ }^{2,5-11}$ presented a robust scheme to calculate distributed polarizabilities. This scheme had the significant advantage of being stable for different choices of basis set. In later publications the approach was referred to as topologically partitioned electric properties (TPEP). It has been applied to calculate intermolecular interaction energies of homomolecular dimers $\mathrm{H}_{2} \mathrm{O}, \mathrm{CO}$, cyanogen, and urea; ${ }^{12,13}$ to investigate transferability of properties in $n$-alkanes; ${ }^{14}$ and to determine distributed electric multipoles in molecules. ${ }^{15}$ Frequency-dependent distributed polarizabilities were calculated for $\mathrm{CO}, \mathrm{H}_{2} \mathrm{O}$, cyanogen, urea, and benzene

\footnotetext{
a) Present address: Materials Ireland Polymer Research Centre, Physics Department, Trinity College, Dublin 2, Ireland. Electronic mail: inhetpm@tcd.ie

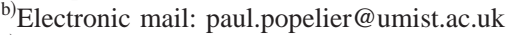

${ }^{c)}$ Electronic mail: r.w.munn@umist.ac.uk

${ }^{\mathrm{d})}$ Electronic mail: angyan@1ctn.uhp-nancy.fr
}

molecules. ${ }^{16}$ The approach was also used to devise a polarizable potential model used in liquid $\mathrm{CCl}_{4}$ simulations. ${ }^{17}$

In this paper we calculate distributed polarizabilities to obtain chemical and physical information about electrostatic properties of the water dimer. Water clusters are of considerable practical significance, and experimental, and theoretical understanding of their behavior has developed greatly in recent years. ${ }^{18-22}$ We seek to enhance that understanding, while also showing the power of distributed polarizabilities to probe aspects of molecular response.

The general theory is described in Sec. II. This is followed in Sec. III by the procedure used to calculate distributed polarizabilities. Results for the water molecule and for the water dimer are given in Sec. IV, and our conclusions are presented in Sec. V. A detailed derivation of the distributed polarizability equation is given in the Appendix.

\section{THEORY}

The general distributed polarizabilities ${ }^{2}$ relate the change in the regular spherical harmonic component $l m$ of the electron density in atomic region $A$ to the regular spherical harmonic component $l^{\prime} m^{\prime}$ of the electric potential in region $B$. The distributed multipole polarizabilities $\alpha_{l m, l^{\prime} m^{\prime}}^{A B}$, have been defined in the CPHF approximation in Ref. 2, whose expression is derived in the Appendix, starting from tensor trace formalism. ${ }^{8}$ The distributed multipole polarizability is given by

$\alpha_{l m, l^{\prime} m^{\prime}}^{A B}=4 \sum_{i \sigma} \sum_{j \tau}\left\langle i\left|\hat{Q}_{l m}\right| \sigma\right\rangle_{A}\left(\mathbf{G}^{-1}\right)_{i \sigma, j \tau}\left\langle j\left|\hat{Q}_{l^{\prime} m^{\prime}}\right| \tau\right\rangle_{B}$,

where $\hat{Q}_{l m}$ is a multipole moment operator defined in regular spherical harmonics, $i$ and $j$ denote occupied molecular orbitals (MO) and $\sigma$ and $\tau$ are virtual molecular orbitals obtained through closed-shell Hartree-Fock calculations. The quantities $\left\langle i\left|\hat{Q}_{l m}\right| \sigma\right\rangle_{A}$ are transition multipole moment ma- 
trix elements in an atomic basis, as defined in the AIM context, with $\mathbf{r}^{A}$ as origin. The matrix $\mathbf{G}$ is defined in terms of two-electron integrals

$$
G_{i \sigma, j \tau}=4(\sigma i \mid j \tau)-(\sigma j \mid i \tau)-(\sigma \tau \mid i j)+\delta_{i j} \delta_{\sigma \tau}\left(\epsilon_{\sigma}-\epsilon_{i}\right),
$$

where $\epsilon_{\sigma}$ and $\epsilon_{i}$ are orbital energies. Here the two-electron part in the $\mathbf{G}$ matrix refers to integrals evaluated after the self-consistent field equations are transformed such that the unperturbed self-consistent field operator is diagonal. ${ }^{9}$ The $\mathbf{G}$ matrix in Eq. (2) differs from that given in standard textbooks. ${ }^{23}$

The transition multipole moments are calculated using the vector $\mathbf{r}^{A}$ in an axis system with the center of mass as origin. All multipole moments are invariant under translation of the molecule, but not under rotation (except for the charge, being a scalar), and hence depend on the choice of axes. This becomes particularly important if one wants to compare properties of a molecule in the dimer with those of the single isolated molecule. The distributed polarizability terms in principle give any arbitrary multipole response of the system to any arbitrary distribution of external potential. As such, they can convey a large amount of information that needs to be processed in order to compare it with experiment or appreciate it properly. In practice, we have restricted ourselves here to charge and dipole response to an external electrostatic potential and field.

\section{A. Polarizability}

Atomic polarizabilities are obtained as ${ }^{2}$

$$
\alpha_{\alpha \beta}^{A}=\sum_{B}\left(r_{\alpha}^{A} \alpha_{q q}^{A B} r_{\beta}^{B}+r_{\alpha}^{A} \alpha_{q \beta}^{A B}+\alpha_{\alpha q}^{A B} r_{\beta}^{B}+\alpha_{\alpha \beta}^{A B}\right),
$$

and then the total molecular dipole polarizability in a uniform electric field (which is the quantity usually reported as the molecular polarizability) can be calculated according to ${ }^{1}$

$$
\begin{aligned}
\alpha_{\alpha \beta} & =\sum_{A} \alpha_{\alpha \beta}^{A} \\
& =\sum_{A} \sum_{B}\left(r_{\alpha}^{A} \alpha_{q q}^{A B} r_{\beta}^{B}+r_{\alpha}^{A} \alpha_{q \beta}^{A B}+\alpha_{\alpha q}^{A B} r_{\beta}^{B}+\alpha_{\alpha \beta}^{A B}\right) .
\end{aligned}
$$

Here the regular spherical harmonic notation has been replaced by one in which subscript $q$ in the first position denotes a charge and in the second position denotes a potential (through which the charge affects the energy). Greek subscripts $\alpha$ and $\beta$ in the first position denote Cartesian components of the dipole moment and in the second position denote Cartesian components of the electric field (through which the dipole moment affects the energy). Then to construct the total molecular dipole polarizability the atomic charge and atomic dipole terms $\alpha_{q q}^{A B}, \alpha_{q \beta}^{A B}, \alpha_{\alpha q}^{A B}, \alpha_{\alpha \beta}^{A B}$ are required. It is not just the atomic dipole moments that contribute to the molecular dipole moment, but also the atomic charges. Each can be affected by a uniform electric field, which corresponds to a linear variation of potential and hence implies different potentials at different atoms. In this context, the charge-potential terms $\alpha_{q q}^{A B}$ can be regarded as mutual capacitances for $A \neq B$ and self-capacitances for $A=B$.
It should be noted that the atomic polarizabilities defined in Eq. (3) lack some expected properties of polarizabilities, since they are not symmetric under interchange of Cartesian components $\alpha$ and $\beta$, and they depend on the choice of origin within the molecule (though not on the choice of origin within each atomic basin). The lack of symmetry arises because the atomic polarizability cannot be obtained as the second derivative of an energy with respect to electric field, basically because response to electric field and potential are treated independently. The origin dependence arises because the dipole moment of a charged species depends on the choice of origin, and the atoms acquire charges from the flows between atomic basins. However, these problems disappear when the total polarizability of the whole neutral system is constructed from the atomic polarizabilities through Eq. (4). Hence the atomic polarizabilities should be regarded as auxiliary quantities.

Similar considerations apply when one divides the total polarizability of the water dimer into molecular polarizabilities. Because there is a net transfer of charge between the molecules, they each acquire a charge, and hence their polarizabilities each depend on the choice of origin. Such molecular polarizabilities can be defined from Eq. (4) by dividing the sum over atoms $A$ into two sums, one over atoms $A \in$ Molecule 1 and the other over atoms $A \in$ Molecule 2 . These evidently sum to the total polarizability of the dimer, but only if the same origin is used for each molecule, whereas it would be natural for comparison with calculations on the separate molecules to take a different origin in each molecule (e.g., the center of mass). Starting from Eq. (4) one can also analyze each molecular polarizability into two parts, one local to a particular molecule, i.e., where atoms $A$ and $B$ both belong to the same molecule, and the other nonlocal between the two molecules, i.e., where atoms $A$ and $B$ belong to different molecules. In this case, the local part is symmetric and the nonlocal part is not, but the nonlocal parts for the two molecules are transposes of one another as required to make the total dimer polarizability symmetric. Again, for these parts to sum to the total dimer polarizability, they must be referred to the same origin.

\section{B. External electric field}

The electric field is obtained as minus the gradient of the potential $V(\mathbf{r})$. Thus a uniform electric field of magnitude $V^{\prime}$ along the $y$ axis is described by the potential

$$
V=-V^{\prime} y \text {, }
$$

where the potential at the origin is taken as zero.

\section{Charge flow}

Net charge flow into a region $A$ is calculated from the expression

$$
\Delta Q_{q}^{A} \equiv \Delta Q_{00}^{A}=\sum_{B} \sum_{l m} \alpha_{00, l m}^{A B} X_{l m}^{B},
$$

suitably converted from regular spherical harmonics to Cartesian components. Thus $X_{00}^{B}=V\left(\mathbf{r}^{B}\right)$ is the electrostatic potential in the origin of atomic basin $B$ and $X_{1 x}^{B}=E_{x}\left(\mathbf{r}^{B}\right)$ is the 
TABLE I. Cartesian atomic coordinates of a single water molecule (equilibrium structure) and of water molecules 1 and 2 in the dimer (global minimum, linear structure). The numbers in brackets refer to the numbering of the atoms in Fig. 1. $\angle \mathrm{HOH}$ denotes the bond angle.

\begin{tabular}{lllrr}
\hline \hline \multicolumn{1}{c}{ Atom } & x/a.u. & y/a.u. & \multicolumn{1}{c}{$z /$ a.u. } & $\angle \mathrm{HOH}{ }^{\circ}$ \\
\hline Single & & & & 104.52 \\
$\mathrm{O}$ & 0.0 & 0.0 & 0.22144 & \\
$\mathrm{H}$ & 0.0 & 1.43047 & -0.88575 & \\
$\mathrm{H}$ & 0.0 & -1.43047 & -0.88575 & \\
Dimer Molecule 1 & & & & 106.10 \\
$\mathrm{O}(1)$ & 0.01508 & -2.71425 & 0.0 & \\
$\mathrm{H}(2)$ & 0.75768 & -3.48995 & 1.42758 & \\
$\mathrm{H}(3)$ & 0.75768 & -3.48995 & -1.42758 & \multirow{2}{*}{105.91} \\
Dimer Molecule 2 & & & & \\
$\mathrm{O}(4)$ & 0.01508 & 2.98988 & 0.0 & \\
$\mathrm{H}(5)$ & -0.08650 & 1.19946 & 0.0 & \\
$\mathrm{H}(6)$ & -1.67008 & 3.57543 & 0.0 & \\
\hline \hline
\end{tabular}

$x$ component of the electric field at the origin of atomic basin $B$. In this paper, "charge"' always refers to positive charge, so that charge flow is opposite in direction to electron flow.

\section{Dipole moment}

The dipole moment $\boldsymbol{\mu}$ is calculated using the electrostatic moments as defined in $\mathrm{AIM}^{4}$

$$
\boldsymbol{\mu}=\sum_{A}\left[q(A) \mathbf{R}_{A}+\mathbf{M}(A)\right]=\boldsymbol{\mu}_{\mathrm{CT}}+\boldsymbol{\mu}_{\mathrm{atom}},
$$

where $\mathbf{R}_{A}$ are the nuclear positions of the atoms, $q(A)$ the atomic charges and $\mathbf{M}(A)$ the atomic dipoles. The molecular dipole moment is the sum of the charge transfer term $\boldsymbol{\mu}_{\mathrm{CT}}$ and the intra-atomic dipole term $\boldsymbol{\mu}_{\text {atom }}$.

\section{CALCULATION DETAILS}

The information in Ref. 2 on how to perform these calculations is very concise. Here we describe the procedure by which we obtain the polarizabilities. In order to calculate the distributed polarizability using Eq. (1), the matrix elements of the transition multipole moments in each atomic basin $\left\langle i\left|\hat{Q}_{l m}\right| \sigma\right\rangle_{A}$ and the elements of the inverse of the twoelectron matrix $G_{i \sigma, j \tau}$ are required.

The starting point is optimization of the geometry of the configuration using an ab initio package such as GAUSSIAN 94. ${ }^{24}$ Using the optimized geometry a wave function is generated. However, as already explained, the two-electron part of the $\mathbf{G}$ matrix requires integrals evaluated after the selfconsistent field equations are transformed such that the unperturbed self-consistent field operator is diagonal (see Sec. II). To achieve this, GAUSSIAN 94 has to be adapted to give a wave function that includes the virtual one-electron molecular orbitals with the same number of coefficients as the occupied orbitals. This nonstandard route (through additional overlays) ensures that the transformed two-electron integrals and orbital energies can be obtained.

The wave function is analyzed using MORPHY01 to identify the atomic basins and to perform the necessary integrations with a high precision. This program ${ }^{25}$ includes a suitable partitioning method based on Atoms in Molecules theory. ${ }^{26,27}$ It, too, is adapted in order to generate the transition multipole moments. Finally, previous techniques ${ }^{28}$ are

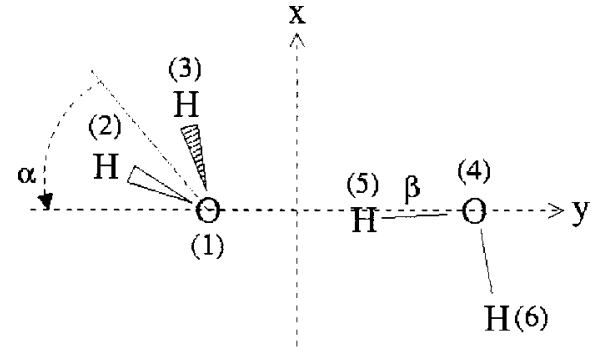

molecule 1

FIG. 1. Water dimer in the global minimum (linear structure) energy geometry (Ref. 32). Numbers in brackets indicate atom numbers; $\alpha$ is the angle between the principal axis of Molecule 1 and the negative $y$ axis; and $\beta$ is the angle between the $\mathrm{O}-\mathrm{H}$ bond (atoms 4 and 5) and the positive $y$ axis. Note that $x y$ is a plane of symmetry for Molecule 1, so that $\mathrm{H}(2)$ and $\mathrm{H}(3)$ have the same $x$ and $y$ coordinates but equal and opposite $z$ coordinates (cf. Table I).

used to process the information from GAUSSIAN $94^{24}$ into a form suitable for use in MORPHY01. ${ }^{25}$ The 6-311+ $+\mathrm{G}(2 d, 2 p)$ and Sadlej ${ }^{29}$ basis sets were used in the calculations.

We have used the Atoms in Molecules ${ }^{3,4}$ partitioning scheme since it has been shown that it yields nearly basis set independent distributed polarizabilities. ${ }^{2}$ Hilbert-space partitioning schemes such as Mulliken, distributed multipole analysis (DMA), and smoothed Gauss-Hermite partitioning lead to unphysically large distributed polarizabilities.,30 Other partitioning schemes such as Stewart Atoms ${ }^{31}$ may have technical advantages but have not hitherto been employed to calculate distributed polarizabilities. A comparison between calculated multipoles in water clusters using AIM and Voronoi partitioning schemes found different molecular dipole moments for the water monomers. ${ }^{18}$

In this paper polarizabilities are quoted in atomic units (a.u.). The atomic unit of the distributed dipole-dipole polarizability is equal to $4 \pi \epsilon_{0} a_{0}^{3}$, with $a_{0}$ the Bohr radius, i.e., $0.1648778 \times 10^{-40} \mathrm{~J}^{-1} \mathrm{C}^{2} \mathrm{~m}^{2}$. In electrostatic units $4 \pi \epsilon_{0}$ is simply unity and 1 a.u. of dipole-dipole polarizability is equal to $0.1481847 \AA^{3}$.

\section{RESULTS}

Atomic and molecular properties have been calculated for a single water molecule and for the water dimer using the structural parameters given in Table I and the global minimum geometry for the dimer illustrated in Fig. 1. Calculations have been performed for the whole interacting dimer system, and also for what we call the "noninteracting

TABLE II. Comparison between calculated and experimental (Ref. 33) (Expt.) global minimum geometries for the water dimer. $R_{\mathrm{OO}}$ is the distance between oxygen atoms, and $\alpha$ and $\beta$ are the angles indicated in Fig. 1. Calculated geometries used GAUSSIAN 94 (Ref. 24) with the 6-311+ $+\mathrm{G}(2 d, 2 p)$ basis set $(\mathrm{G})$ or Sadlej basis set (Ref. 29) (Sadlej) or the anisotropic site potential (Ref. 32) (ASP).

\begin{tabular}{lcccc}
\hline \hline & $\mathrm{G}$ & Sadlej & ASP & Expt. \\
\hline$R_{\mathrm{OO}}$ /a.u. & 5.74 & 5.70 & 5.64 & $5.50 \pm 0.01$ \\
$\alpha /^{\circ}$ & 49 & 44 & 63 & $57 \pm 10$ \\
$\beta / /^{\circ}$ & 3 & 3 & 0 & $5 \pm 10$ \\
\hline \hline
\end{tabular}


TABLE III. Components of the distributed polarizability of a single water molecule in a.u. using precise MORPHY integration. Note that this table allows reconstruction of complete matrices for all atom combinations, since $\alpha_{\alpha \beta}^{\mathrm{O} 1-\mathrm{H} 2}=\alpha_{\beta \alpha}^{\mathrm{H} 2-\mathrm{O} 1}$ and so on.

\begin{tabular}{|c|c|c|c|c|c|c|c|c|c|c|c|c|c|c|c|c|}
\hline & $q q$ & $q x$ & $q y$ & $q z$ & $x q$ & $y q$ & $z q$ & $x x$ & $x y$ & $x z$ & $y x$ & $y y$ & $y z$ & $z x$ & $z y$ & $z z$ \\
\hline $\mathrm{O} 1-\mathrm{O} 1$ & 0.738 & 0.000 & 0.000 & 0.219 & 0.000 & 0.000 & 0.219 & 6.873 & 0.000 & 0.000 & 0.000 & 4.048 & 0.000 & 0.000 & 0.000 & 5.137 \\
\hline $\mathrm{O} 1-\mathrm{H} 2$ & -0.369 & -0.000 & -0.207 & 0.171 & -0.000 & 0.136 & -0.110 & 0.166 & -0.000 & 0.000 & -0.000 & 0.114 & -0.018 & 0.000 & 0.000 & 0.187 \\
\hline $\mathrm{O} 1-\mathrm{H} 3$ & -0.369 & -0.000 & 0.207 & 0.171 & -0.000 & -0.136 & -0.110 & 0.166 & 0.000 & 0.000 & 0.000 & 0.114 & 0.018 & 0.000 & -0.000 & 0.187 \\
\hline $\mathrm{H} 2-\mathrm{H} 2$ & 0.410 & 0.000 & 0.233 & -0.194 & 0.000 & 0.233 & -0.194 & 0.123 & 0.000 & 0.000 & 0.000 & 0.243 & -0.116 & 0.000 & -0.116 & 0.227 \\
\hline $\mathrm{H} 2-\mathrm{H} 3$ & -0.041 & 0.000 & 0.026 & 0.023 & 0.000 & -0.026 & 0.023 & -0.001 & 0.000 & 0.000 & 0.000 & 0.018 & 0.013 & 0.000 & -0.013 & -0.014 \\
\hline H3-H3 & 0.410 & 0.000 & -0.233 & -0.194 & 0.000 & -0.233 & -0.194 & 0.123 & 0.000 & 0.000 & 0.000 & 0.243 & 0.116 & 0.000 & 0.116 & 0.227 \\
\hline
\end{tabular}

dimer." The noninteracting dimer calculations are performed on two separate single water molecules but using the geometry of the two molecules in the interacting dimer and the same system of axes. This allows us to isolate those features that arise directly from the interaction in the dimer (and hence by implication are mediated by the hydrogen bond) from those that arise from the concomitant change in geometry from the single molecule. Table I shows the optimized water dimer coordinates using a Sadlej basis set ${ }^{29}$ starting from the global minimum geometry (linear structure). ${ }^{32}$ Table II compares various theoretical calculated values and the experimental results for the oxygen-oxygen distance $R_{\mathrm{OO}}$ and angles $\alpha$ and $\beta$ (see Fig. 1). The optimized geometry using $6-311++\mathrm{G}(2 d, 2 p)$ and Sadlej ${ }^{29}$ basis sets underestimates $R_{\mathrm{OO}}$ and $\alpha$, and overestimates $\beta$ compared with Millot and Stone, ${ }^{32}$ but is in reasonable agreement with experiment. ${ }^{33}$

\section{A. Water monomer}

The full set of TPEP distributed polarizabilities obtained for the water monomer with a Sadlej basis set ${ }^{29}$ are displayed in Table III. Here $q q$ refers to $\alpha_{q q}^{A B}$, the charge-charge term (which is independent of the choice of origin); $q x, q y$, and $q z$ refer to the vector components $\alpha_{q \beta}^{A B}$ (which are equivalent to the components $\alpha_{\beta q}^{B A}$ ); and $x x, x y, \ldots, z z$ refer to the components of the tensor $\alpha_{\alpha \beta}^{A B}$ (which are equivalent to $\alpha_{\beta \alpha}^{B A}$ ). Our values are within $1.5 \%$ of those calculated in Ref. 2 at the same basis set level. Differences arise from differences in the precision of the integration of the transition multipole moments. Our integration errors are smaller than 1.7 $\times 10^{-4}$ a.u. For comparison, the total polarizability components for the water monomer in the equilibrium geometry calculated from the TPEP distributed polarizabilities are

TABLE IV. Comparison between experimental (Ref. 48) and calculated polarizability components of a single water molecule in the equilibrium geometry; $\bar{\alpha}$ denotes the average polarizability, $\frac{1}{3} \operatorname{Tr} \alpha$. TPEP indicates polarizability components reconstructed from distributed polarizability components using precise MORPHY integration. HF, MP2, and B3LYP indicate GAUSSIAN 94 (Ref. 24) calculations at different levels. All calculations used a Sadlej basis set (Ref. 29).

\begin{tabular}{lcccc}
\hline \hline Method & $\alpha_{x x}$ /a.u. & $\alpha_{y y} /$ a.u. & $\alpha_{z z} /$ a.u. & $\bar{\alpha} /$ a.u. \\
\hline TPEP & 7.78 & 9.14 & 8.46 & 8.46 \\
HF & 7.82 & 9.17 & 8.49 & 8.49 \\
MP2 & 9.46 & 9.85 & 9.56 & 9.62 \\
B3LYP & 9.49 & 10.08 & 9.75 & 9.77 \\
Exp. & 9.91 & 10.31 & 9.55 & 9.92 \\
\hline \hline
\end{tabular}

shown in Table IV, together with the experimental result. Self-consistent field (SCF) calculations at Hartree-Fock level lie within $15 \%$ of experiment, whereas more sophisticated methods such as second-order Møller-Plesset Becke3Lee-Yang-Parr and (B3LYP) (MP2) reproduce experiment within $3 \%$.

The atomic and molecular polarizabilities constructed from the distributed components using Eqs. (3) and (4) are shown in Table $\mathrm{V}$, which should be read in conjunction with the structural information in Table I. The molecular polarizability is just the sum of the atomic terms, cf. Eq. (4). The table shows clearly that the oxygen atom is more polarizable than the hydrogen atoms. The polarizability components naturally also reflect the geometry, with the $\mathrm{OH}$ bond lying in the $y z$ plane. The reconstructed total polarizability (the TPEP value in Table IV) is in very good agreement with the CPHF result from the GAUSSIAN 94 program. The reason why the agreement is not perfect can be traced to the following technical point.

As a result of conservation of charge, the atomic overlap integral matrix elements (AOMs) $\langle i \mid \sigma\rangle_{\mathrm{A}}$ should satisfy $\Sigma_{A}\langle i \mid \sigma\rangle_{A}=0$, where the summation runs over all $N$ atomic basins. This relation is also a trivial consequence of the orthogonality of the molecular orbitals. Unfortunately, for the numerically integrated transition densities the relation is violated to an extent that depends on the truncation errors in the numerical integration. One treatment to restore the orbital orthogonality is to calculate the total error $\epsilon$ for each set of AOMs as $\Sigma_{A}\langle i \mid \sigma\rangle_{A}=\epsilon$ and then correct each AOM by dividing the error uniformly between them all, so that $\langle i \mid \sigma\rangle_{A}$ $\rightarrow\langle i \mid \sigma\rangle_{A}-\epsilon / N$. As an example, for the water monomer calculation with a Sadlej basis set using a standard MORPHY integration procedure, the largest error for the AOMs is found to be $2 \times 10^{-2}$. Applying the correction scheme reduces this error to $9 \times 10^{-8}$, which increases the TPEP mean polarizability from $\bar{\alpha}=8.44$ a.u. before correction to $\bar{\alpha}$ $=8.46$ a.u. after correction, so improving the agreement be-

TABLE V. Atomic and molecular polarizability in a.u. of a single water molecule in the equilibrium geometry, using a Sadlej basis set (Ref. 29). In this case the total polarizability is the sum of the atomic components.

\begin{tabular}{lccccrc}
\hline \hline & $x x$ & $x y$ & $x z$ & $y y$ & \multicolumn{1}{c}{$y z$} & $z z$ \\
\hline O1 & 7.204 & 0.000 & 0.000 & 4.665 & 0.000 & 5.925 \\
H2 & 0.288 & 0.000 & 0.000 & 2.235 & -1.314 & 1.267 \\
H3 & 0.288 & 0.000 & 0.000 & 2.235 & 1.314 & 1.267 \\
Total & 7.780 & 0.000 & 0.000 & 9.136 & 0.000 & 8.459 \\
\hline \hline
\end{tabular}


TABLE VI. Charge flow in a single water molecule and in the interacting and noninteracting water dimer under the influence of a uniform external field in the $y$ direction. The entries in the table are expressed as charge flow $\Delta Q /$ a.u. per unit field $V^{\prime} /$ a.u.

\begin{tabular}{lccrrr}
\hline \hline \multicolumn{1}{c}{ Structure } & Molecule & $\Delta Q_{q}^{\mathrm{O}}$ & \multicolumn{1}{c}{$\Delta Q_{q}^{\mathrm{H} 1}$} & \multicolumn{1}{c}{$\Delta Q_{q}^{\mathrm{H} 2}$} & \multicolumn{1}{c}{ Total } \\
\hline Single molecule & & 0.0 & 0.252 & -0.252 & \multicolumn{1}{c}{0.0} \\
Interacting dimer & 1 & 0.001 & -0.156 & -0.156 & -0.310 \\
& 2 & 0.414 & -0.270 & 0.168 & 0.312 \\
Noninteracting dimer & 1 & 0.161 & -0.081 & -0.081 & -0.001 \\
& 2 & 0.133 & -0.256 & 0.127 & 0.001 \\
\hline \hline
\end{tabular}

tween the TPEP and the HF values. Alternatively, a more precise MORPHY integration reduces the largest error for the AOMs to about $10^{-8}$ (which is the highest numerical precision accessible to us), leading to the same value of $\bar{\alpha}$ $=8.46$ a.u. before any corrections.

An application of the distributed polarizability components is to investigate charge flow in a molecule under the effect of arbitrary external fields. Using Eq. (6) with a simple uniform external field in the $x$ direction normal to the molecular plane shows no charge flow between any of the atoms. An external field in the $y$ direction causes charge to flow from hydrogen atom 2 to atom 1, as reported in Table VI, while an external field in the positive $z$ direction shifts charge from the hydrogen atoms to the oxygen. By symmetry, an external field causes charge to flow in the $y z$ plane of the molecule, though the total change in charge is of course zero. Note that the changes in electric charge depend on the molecular orientation with respect to the field. Therefore, in order to compare charge flow in similar molecules in different orientations, as in the dimer considered below, the distributed polarizability components need to be transformed into some standard orientation.

\section{B. Water dimer}

The independent distributed polarizability components for the water dimer were calculated following the same computational procedure as for a single water monomer. Our results for the interacting dimer have been compared not only with the single water molecule but also with a dimer composed of noninteracting water molecules. A selected set of results (10 components) is shown in Table VII for the noninteracting dimer and in Table VIII for the interacting dimer. As can be seen, the calculations generate a wealth of data, which is manageable for a simple molecule like water, but for larger systems such as the water dimer starts to become overwhelming: 16 charge and dipole components for each of $\frac{1}{2} N(N+1)$ distinct pairs for $N$ atoms. Therefore it is important to find a suitable way of analyzing the data. Our finding that charge flow occurs mainly along the $\mathrm{O}-\mathrm{H}$ bonds assists this analysis here. The charge-charge flow terms also reveal whether two molecules possess the same molecular geometry, since these terms are invariant under rotation.

The analysis of the distributed polarizability components was conducted in the same manner as for the water monomer. Table IX shows how the atomic charge-charge polarizabilities change from a single water molecule to the molecules in noninteracting and interacting water dimers. This allows the changes due to the geometry change to be separated from those explicitly due to the hydrogen bond interaction. Part (a) of the table shows that the increase in the $\mathrm{OHO}$ angle in the noninteracting dimer molecules generally lowers the distributed polarizability components compared with the single isolated molecule, with broadly the same effect on each molecule. Allowing the molecules in the dimer to interact generally causes a further reduction in polarizability, with significantly different effects on the two molecules. In particular, Molecule 2 exhibits much larger changes due to the interaction than those due to the geometry change, primarily affecting the $\mathrm{O} 4$ and $\mathrm{H} 5$ atoms involved in the hydrogen bond. The largest additional component due to the interaction is that linking the two oxygen atoms, consistent with their connection via the hydrogen bond.

For the higher polarizabilities, such detailed analysis becomes very complex. Table $\mathrm{X}$ shows charge-field polarizabilities between selected pairs of atoms in a single water molecule and in noninteracting and interacting water dimers, in each case transformed to the standard molecular axes. Once again, the geometry change leaves the two noninteracting molecules with very similar responses, whereas the interaction differentiates much more between the two molecules.

The atomic and molecular polarizabilities were calcu-

TABLE VII. Selected components of the distributed polarizability in a.u. of the noninteracting water dimer. Atom labels refer to coordinates in Table I. Thus $\mathrm{O} 1, \mathrm{H} 2, \mathrm{H} 3$ refer to Molecule 1 and O4, H5, and H6 refer to Molecule 2. Because the molecules are not interacting, no components relate atoms in different molecules.

\begin{tabular}{|c|c|c|c|c|c|c|c|c|c|c|}
\hline & $q q$ & $q x$ & $q y$ & $q z$ & $x x$ & $x y$ & $x z$ & $y y$ & $y z$ & $z z$ \\
\hline O1-O1 & 0.705 & -0.145 & 0.152 & 0.000 & 6.037 & 0.832 & 0.000 & 5.965 & 0.000 & 4.010 \\
\hline $\mathrm{O} 1-\mathrm{H} 2$ & -0.353 & -0.112 & 0.117 & -0.201 & 0.168 & -0.013 & 0.000 & 0.169 & 0.000 & 0.112 \\
\hline O1-H3 & -0.353 & -0.112 & 0.117 & 0.201 & 0.168 & -0.013 & 0.000 & 0.169 & 0.000 & 0.112 \\
\hline $\mathrm{H} 2-\mathrm{H} 2$ & 0.390 & 0.126 & -0.132 & 0.225 & 0.158 & -0.049 & 0.078 & 0.162 & -0.081 & 0.233 \\
\hline $\mathrm{H} 2-\mathrm{H} 3$ & -0.038 & -0.014 & 0.015 & 0.024 & -0.007 & 0.006 & 0.008 & -0.007 & -0.009 & 0.017 \\
\hline $\mathrm{H} 3-\mathrm{H} 3$ & 0.390 & 0.126 & -0.132 & -0.225 & 0.158 & -0.049 & -0.078 & 0.162 & 0.081 & 0.233 \\
\hline O4-O4 & 0.709 & 0.175 & 0.118 & 0.000 & 4.804 & 0.531 & 0.000 & 4.366 & 0.000 & 6.833 \\
\hline O4-H5 & -0.358 & 0.024 & 0.260 & 0.000 & 0.151 & 0.025 & 0.000 & 0.143 & 0.000 & 0.158 \\
\hline O4-H6 & -0.351 & 0.246 & -0.076 & 0.000 & 0.171 & 0.038 & 0.000 & 0.123 & 0.000 & 0.156 \\
\hline H5-H5 & 0.396 & -0.027 & -0.291 & 0.000 & 0.116 & 0.033 & 0.000 & 0.333 & 0.000 & 0.114 \\
\hline H5-H6 & -0.038 & 0.031 & -0.009 & 0.000 & -0.004 & -0.002 & 0.000 & 0.008 & 0.000 & -0.001 \\
\hline H6-H6 & 0.389 & -0.276 & 0.085 & 0.000 & 0.320 & -0.054 & 0.000 & 0.121 & 0.000 & 0.110 \\
\hline
\end{tabular}


TABLE VIII. Selected components of the distributed polarizability in a.u. of the interacting water dimer. Atom labels refer to coordinates in Table I. Because the molecules are interacting, nonzero components relate atoms in different molecules, i.e., 1, 2, and 3 to 4,5 , and 6.

\begin{tabular}{|c|c|c|c|c|c|c|c|c|c|c|}
\hline & $q q$ & $q x$ & $q y$ & $q z$ & $x x$ & $x y$ & $x z$ & $y y$ & $y z$ & $z z$ \\
\hline $\mathrm{O} 1-\mathrm{O} 1$ & 0.711 & -0.151 & 0.018 & 0.000 & 5.732 & 0.734 & 0.000 & 4.780 & 0.000 & 3.826 \\
\hline $\mathrm{O} 1-\mathrm{H} 2$ & -0.331 & -0.103 & 0.108 & -0.185 & 0.158 & 0.011 & 0.000 & 0.146 & 0.014 & 0.108 \\
\hline $\mathrm{O} 1-\mathrm{H} 3$ & -0.331 & -0.103 & 0.108 & 0.185 & 0.158 & 0.011 & 0.000 & 0.146 & -0.014 & 0.108 \\
\hline $\mathrm{O} 1-\mathrm{O} 4$ & -0.041 & 0.009 & 0.002 & 0.000 & -0.068 & -0.005 & 0.000 & 0.069 & 0.000 & -0.063 \\
\hline $\mathrm{O} 1-\mathrm{H} 5$ & -0.003 & -0.001 & 0.000 & 0.000 & 0.033 & 0.004 & 0.000 & 0.074 & 0.000 & 0.034 \\
\hline O1-H6 & -0.005 & 0.004 & -0.001 & 0.000 & -0.018 & 0.002 & 0.000 & 0.008 & 0.000 & -0.004 \\
\hline $\mathrm{H} 2-\mathrm{H} 2$ & 0.377 & 0.119 & -0.126 & 0.214 & 0.146 & -0.046 & 0.072 & 0.152 & -0.076 & 0.218 \\
\hline $\mathrm{H} 2-\mathrm{H} 3$ & -0.035 & -0.013 & 0.013 & 0.022 & -0.006 & 0.006 & 0.007 & -0.006 & -0.007 & 0.015 \\
\hline $\mathrm{H} 2-\mathrm{O} 4$ & -0.011 & -0.009 & -0.016 & -0.019 & -0.007 & -0.006 & -0.005 & 0.009 & 0.004 & -0.013 \\
\hline $\mathrm{H} 2-\mathrm{H} 5$ & 0.002 & -0.001 & 0.001 & -0.001 & -0.001 & 0.000 & 0.000 & 0.000 & 0.000 & -0.001 \\
\hline $\mathrm{H} 2-\mathrm{H} 6$ & -0.002 & 0.002 & -0.001 & 0.000 & 0.000 & 0.000 & 0.000 & 0.001 & 0.000 & 0.000 \\
\hline $\mathrm{H} 3-\mathrm{H} 3$ & 0.377 & 0.119 & -0.126 & -0.214 & 0.146 & -0.046 & -0.072 & 0.152 & 0.076 & 0.218 \\
\hline $\mathrm{H} 3-\mathrm{O} 4$ & -0.011 & -0.009 & -0.016 & 0.019 & -0.007 & -0.006 & 0.005 & 0.009 & -0.004 & -0.013 \\
\hline H3-H5 & 0.002 & -0.001 & 0.001 & 0.001 & -0.001 & 0.000 & 0.000 & 0.000 & 0.000 & -0.001 \\
\hline $\mathrm{H} 3-\mathrm{H} 6$ & -0.002 & 0.002 & -0.001 & 0.000 & 0.000 & 0.000 & 0.000 & 0.001 & 0.000 & 0.000 \\
\hline $\mathrm{O} 4-\mathrm{O} 4$ & 0.676 & 0.182 & 0.123 & 0.000 & 4.885 & 0.557 & 0.000 & 4.482 & 0.000 & 7.037 \\
\hline O4-H5 & -0.244 & 0.013 & 0.113 & 0.000 & 0.092 & 0.009 & 0.000 & 0.075 & 0.000 & 0.093 \\
\hline O4-H6 & -0.368 & 0.260 & -0.079 & 0.000 & 0.176 & 0.038 & 0.000 & 0.127 & 0.000 & 0.166 \\
\hline H5-H5 & 0.267 & -0.013 & -0.129 & 0.000 & 0.051 & 0.008 & 0.000 & 0.082 & 0.000 & 0.050 \\
\hline H5-H6 & -0.026 & 0.021 & -0.006 & 0.000 & -0.003 & -0.002 & 0.000 & 0.003 & 0.000 & 0.000 \\
\hline H6-H6 & 0.404 & -0.289 & 0.089 & 0.000 & 0.339 & -0.055 & 0.000 & 0.131 & 0.000 & 0.121 \\
\hline
\end{tabular}

lated using Eqs. (3) and (4). Table XI displays the molecular polarizability for each of the monomers in the dimer. Calculating the molecular polarizabilities of two or more interacting molecules requires a treatment of distributed response, and hence is not possible with standard ab initio implementations such as GAUSSIAN 94. Using distributed polarizabilities to calculate individual molecular polarizabilities in a set of molecules is then clearly a useful tool, since it allows one to investigate the change in polarizability when the interaction between molecules is turned on. In addition to this, the atomic polarizabilities provide a powerful tool for investigating which section of a large molecule is more susceptible to electron movements under the influence of an external field.

TABLE IX. Changes in atomic charge-charge polarizabilities $\alpha_{q q}^{A B} /$ a.u: (a) in the noninteracting dimer relative to a single water molecule, thereby showing the effect of the geometry changes in the dimer; (b) in the interacting dimer relative to the noninteracting dimer, thereby showing the additional effect of the interaction. As the polarizability matrix is symmetric under interchange of atoms $A$ and $B$, only the upper triangle is shown; by definition, the geometry effect alone induces no interactions between the molecules.

\begin{tabular}{|c|c|c|c|c|c|c|}
\hline Atom $A$ : & $\mathrm{O} 1$ & $\mathrm{H} 2$ & $\mathrm{H} 3$ & $\mathrm{O} 4$ & H5 & H6 \\
\hline Atom $B$ & \multicolumn{6}{|c|}{ (a) Geometry effect } \\
\hline O1 & \multirow[t]{6}{*}{-0.032} & 0.016 & 0.016 & 0 & 0 & 0 \\
\hline $\mathrm{H} 2$ & & -0.020 & 0.003 & 0 & 0 & 0 \\
\hline $\mathrm{H} 3$ & & & -0.020 & 0 & 0 & 0 \\
\hline $\mathrm{O} 4$ & & & & -0.028 & 0.011 & 0.018 \\
\hline H5 & & & & & -0.014 & 0.003 \\
\hline \multirow[t]{2}{*}{ H6 } & & & & & & -0.021 \\
\hline & \multicolumn{6}{|c|}{ (b) Interaction effect } \\
\hline $\mathrm{O} 1$ & 0.006 & 0.022 & 0.022 & -0.041 & -0.003 & -0.005 \\
\hline $\mathrm{H} 2$ & & -0.013 & 0.003 & -0.011 & 0.002 & -0.002 \\
\hline $\mathrm{H} 3$ & & & -0.013 & -0.011 & 0.002 & -0.002 \\
\hline $\mathrm{O} 4$ & & & & -0.033 & 0.114 & -0.017 \\
\hline $\mathrm{H} 5$ & & & & & -0.129 & 0.012 \\
\hline H6 & & & & & & 0.015 \\
\hline
\end{tabular}

Note that although the molecular geometries of the monomers in the noninteracting dimer are the same, the molecular polarizability components are not equal in the dimer axis system, since the monomers are differently aligned in space. Using a suitable transformation confirms that the molecular polarizabilities of the monomers are equal. In contrast, the molecular polarizabilities in the interacting dimer are never equal since the molecules form an unsymmetrical hydrogen bond. The effect of interaction can be seen from the average molecular polarizability (one-third of the trace of the polarizability tensor). For the noninteracting dimer the average is the same for each molecule, namely 8.3 a.u., whereas for the interacting dimer the average is 7.5 a.u. for Molecule 1 and 7.8 a.u. for Molecule 2.

Owing to the formation of the hydrogen bond, both molecules become less polarizable. However, Molecule 1 is affected more than Molecule 2. Comparison of the relative changes shows that the polarizability reduces most signifi-

TABLE X. Selected atomic charge-field polarizability components $\alpha_{q \beta}^{A B} /$ a.u. transformed to the standard geometry of the single water molecule. Single indicates a single water molecule in the equilibrium geometry; dimer 1 and dimer 2 refer to Molecules 1 and 2 in the dimer; non-int and int refer to noninteracting and interacting molecules, respectively.

\begin{tabular}{lcrrc}
\hline \hline & $A-B$ & $q x$ & \multicolumn{1}{c}{$q y$} & $q z$ \\
\hline Single & O1-O1 & -0.006 & 0.000 & 0.214 \\
& O1-H2 & -0.001 & -0.206 & 0.170 \\
Dimer 1 (non-int) & O1-O1 & 0.000 & 0.000 & 0.210 \\
& O1-H2 & 0.000 & -0.201 & 0.162 \\
Dimer 2 (non-int) & O4-O4 & 0.000 & -0.001 & 0.211 \\
& O4-H5 & 0.000 & -0.203 & 0.165 \\
Dimer 1 (int) & O1-O1 & 0.097 & 0.000 & 0.117 \\
\multirow{2}{*}{ Dimer 2 (int) } & O1-H2 & 0.000 & -0.185 & 0.149 \\
& O4-O4 & 0.000 & -0.001 & 0.220 \\
& O4-H5 & 0.000 & -0.087 & 0.074 \\
\hline \hline
\end{tabular}


TABLE XI. Comparison between the molecular polarizability components $\alpha_{\alpha \beta} /$ a.u. in a dimer with interacting molecules and in a dimer with noninteracting molecules.

\begin{tabular}{|c|c|c|c|c|c|c|c|}
\hline \multirow[b]{2}{*}{ Dimer } & \multirow[b]{2}{*}{$\beta$} & \multicolumn{3}{|c|}{ Molecule 1} & \multicolumn{3}{|c|}{ Molecule 2} \\
\hline & & $x$ & $y$ & $z$ & $x$ & $y$ & $z$ \\
\hline \multirow{4}{*}{ Interacting } & $\alpha$ & & & & & & \\
\hline & $x$ & 7.550 & -0.278 & 0.000 & 8.467 & -0.328 & 0.000 \\
\hline & $y$ & -0.278 & 6.556 & 0.000 & -0.328 & 7.333 & 0.000 \\
\hline & $z$ & 0.000 & 0.000 & 8.505 & 0.000 & 0.000 & 7.726 \\
\hline \multirow{3}{*}{ Noninteracting } & $x$ & 7.952 & -0.286 & 0.000 & 8.442 & -0.291 & 0.000 \\
\hline & $y$ & -0.286 & 7.977 & 0.000 & -0.291 & 8.736 & 0.000 \\
\hline & $z$ & 0.000 & 0.000 & 8.896 & 0.000 & 0.000 & 7.683 \\
\hline
\end{tabular}

cantly $(16 \%-18 \%)$ along the direction of the hydrogen bond ( $y$ direction). In the $x$ and $z$ directions Molecule 1 becomes $5 \%$ less polarizable, while Molecule 2 shows a slight increase in polarizability. Hydrogen bond formation reduces the overall polarizability as a result of reorganisation of charge density along the direction of the hydrogen bond. In the dimer Molecule 1 is less polarizable than Molecule 2 and this will obviously manifest itself when the dimer is placed in an external field.

On the other hand, it is well-known that the dipole moment is enhanced in the interacting dimer. ${ }^{18,34,35}$ Dipole moments were calculated using Eq. (7). In the noninteracting dimer, each molecule has a dipole moment of $1.95 \mathrm{D}$, with a net dipole moment for the dimer of 2.51 D. In the interacting dimer, we find that Molecule 1 has a dipole moment of 2.09 $\mathrm{D}$ and Molecule 2 a dipole moment of $2.16 \mathrm{D}$. Because the molecules acquire net charges, these values depend on the choice of origin, which we take as the center of mass of the dimer. The charge transfer between the molecules is 0.01 a.u., which contributes to these dipole moments and thence to the enhancement of the net dipole moment for the dimer, which is $2.97 \mathrm{D}$. Because the moments of the two molecules add vectorially, the net moment in the interacting dimer actually increases by more than the algebraic sum of the increases in the individual molecules. This is consistent with the "co-operative effect" in $\alpha$-helices, whereby it becomes easier to form second and subsequent hydrogen bonds because the molecular dipole moments are enhanced in the process. ${ }^{36,37}$ However, our main finding here is that while the molecules acquire larger dipole moments owing to the formation of the hydrogen bond, they become less polarizable.

Table VI compares the atomic charge flows in both dimers in a uniform field in the $y$ direction. The totals for the noninteracting dimer are not rigorously zero, but only because of finite integration errors. The atomic charge flow reflects the molecular orientation in space. The hydrogens in Molecule 1 differ only in the sign of their $z$ coordinate, and hence respond to an external field in similar fashion, so that charge flows from the hydrogen atoms $\mathrm{H} 2$ and $\mathrm{H} 3$ to the oxygen atom $\mathrm{O} 1$. In Molecule 2 the hydrogens behave differently, so that charge flows from the first hydrogen atom $\mathrm{H} 5$ to $\mathrm{O} 4$ and to the second hydrogen atom H6. In the case of the interacting dimer the total molecular charge flow is nonzero. Charge flows from Molecule 1 to Molecule 2, in the direction of the field. Reversing the direction of the field
TABLE XII. Atomic charge flows in the interacting dimer caused by uniform external fields in the $x, y$ and $z$ directions. The entries in the table are expressed as charge flow $\Delta Q /$ a.u. per unit field $V^{\prime}$ /a.u.

\begin{tabular}{lrcc}
\hline \hline & \multicolumn{3}{c}{ Field direction } \\
Charge flow & \multicolumn{1}{c}{$x$} & $y$ & \multicolumn{1}{c}{$z$} \\
\hline$\Delta Q_{q}^{\mathrm{O} 1}$ & -0.138 & 0.001 & 0.0 \\
$\Delta Q_{q}^{\mathrm{H} 1}$ & 0.088 & -0.156 & 0.241 \\
$\Delta Q_{q}^{\mathrm{H} 2}$ & 0.088 & -0.156 & -0.241 \\
Total, Molecule 1 & 0.038 & -0.310 & 0.0 \\
$\Delta Q_{q}^{\mathrm{O4}}$ & 0.190 & 0.414 & 0.0 \\
$\Delta Q_{q}^{\mathrm{H} 5}$ & 0.022 & -0.270 & 0.0 \\
$\Delta Q_{q}^{\mathrm{H} 6}$ & -0.252 & 0.168 & 0.0 \\
Total, Molecule 2 & -0.040 & 0.312 & 0.0 \\
\hline \hline
\end{tabular}

would have the opposite effect. The atomic charge flow still reflects the molecular orientation.

In the noninteracting dimer oxygen $\mathrm{O} 1$ in Molecule 1 acquires charge from the hydrogen atoms $\mathrm{H} 2$ and $\mathrm{H} 3$. However, in the interacting dimer the charge on $\mathrm{O} 1$ remains essentially unchanged. The oxygen atom gains charge from $\mathrm{H} 2$ and $\mathrm{H} 3$, but immediately loses it to the directly hydrogen bonded atom $\mathrm{H} 5$ in Molecule 2, which in turn loses charge to $\mathrm{O} 4$ and H6. Thus under the influence of an external uniform field in the positive $y$ direction, the hydrogen bond permits charge flow from Molecule 1 to Molecule 2. To demonstrate the connection between charge flow and the hydrogen bond direction, Table XII shows atomic charge flows for uniform fields in the $x, y$, and $z$ directions. Charge flow between the interacting molecules is significant only when the external field is along the direction of the hydrogen bond.

It should be noted that for an accurate calculation of the charge flow using Eq. (6), distributed polarizability components of all combinations of atoms are needed. Contributions of distributed polarizability between atoms as far apart as H2, H3, and H6 might seem insignificant (see Table VIII), but omitting them results in nonzero total charge flow (beyond that attributable to the finite integration errors already mentioned). This can be rationalized because their modest intrinsic response is amplified by the large potential difference that arises from their large separation.

The difference in the behavior of the monomers in the interacting dimer under an external field can also be seen from observing the distributed polarizability components in Table VII and Table VIII. Figure 2 shows the electric field at the atoms for the first and second fields. Charge-charge terms for $\mathrm{O} 1 \mathrm{O} 1$ and $\mathrm{O} 4 \mathrm{O} 4$ are similar for the noninteracting dimer, whereas they differ for the interacting dimer. A similar observation can be made between the $\mathrm{O} 1 \mathrm{H} 2, \mathrm{O} 1 \mathrm{H} 3$, and $\mathrm{O} 4 \mathrm{H} 5$, O4H6 terms. Note that this sort of preliminary analysis is conducted most readily for the charge-charge terms since they are invariant to the molecular orientation in space.

Hitherto we have compared the polarizability of the interacting dimer with that of the noninteracting dimer consisting of two molecules that have the same geometry as in the dimer but are treated entirely separately. It is also possible to compare it with that of the pair of molecules assigned the same noninteracting polarizabilities but allowed to interact purely electrostatically. This allows us to identify changes in 


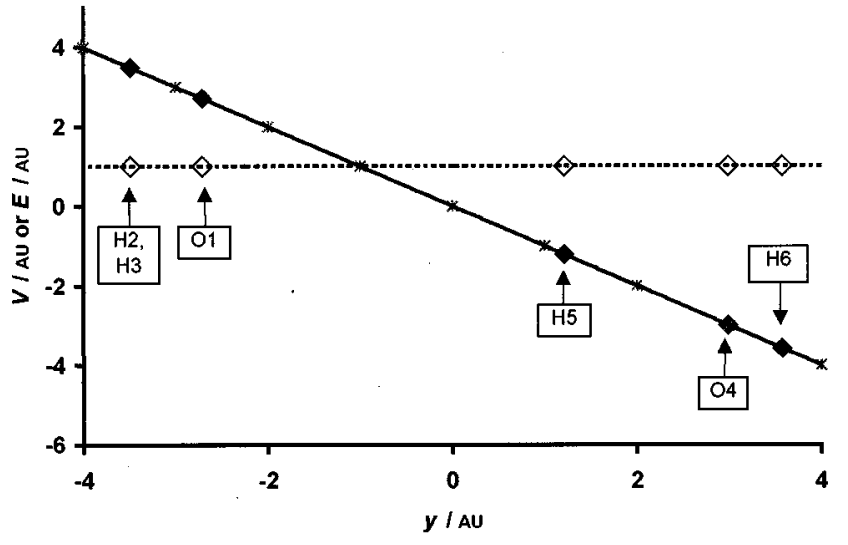

FIG. 2. Model electric potential (filled symbols) when a uniform electric field of 1.0 a.u. (open symbols) is applied to the water dimer along the $y$ axis. Symbols are plotted at the atomic positions in Fig. 1, as identified by the labels.

the dimer response that are not attributable to electrostatic interactions. The essence of the electrostatic treatment was presented long ago for atoms, ${ }^{38}$ and was updated later for anisotropic molecules. ${ }^{39}$ Let the molecules have polarizabilities $\boldsymbol{\alpha}_{1}$ and $\boldsymbol{\alpha}_{2}$ and acquire induced dipole moments $\mathbf{p}_{1}$ and $\mathbf{p}_{2}$ in a uniform external field $\mathbf{E}$. The dipole moments are given by $\boldsymbol{\alpha}_{1} \cdot \mathbf{F}_{1}$ and $\boldsymbol{\alpha}_{2} \cdot \mathbf{F}_{2}$, where $\mathbf{F}_{1}$ and $\mathbf{F}_{2}$ are the local electric fields at the two molecules, given by the external field plus the field produced at the molecule in question by the induced dipole on the other molecule

$$
\mathbf{F}_{1}=\mathbf{E}+\mathbf{T} \cdot \mathbf{p}_{2}, \quad \mathbf{F}_{2}=\mathbf{E}+\mathbf{T} \cdot \mathbf{p}_{1} .
$$

Here $\mathbf{T}$ is the symmetric dipole tensor with Cartesian components given by

$$
T_{\alpha \beta}=\left(3 R_{\alpha} R_{\beta}-R^{2} \delta_{\alpha \beta}\right) / 4 \pi \epsilon_{0} R^{5},
$$

where $\mathbf{R}$ is the vector between the two molecules. Solution for the induced dipole moments yields

$$
\left(\begin{array}{l}
\mathbf{p}_{1} \\
\mathbf{p}_{2}
\end{array}\right)=\left(\begin{array}{ll}
\boldsymbol{\alpha}_{1}^{-1} & -\mathbf{T} \\
-\mathbf{T} & \boldsymbol{\alpha}_{2}^{-1}
\end{array}\right)^{-1}\left(\begin{array}{l}
\mathbf{E} \\
\mathbf{E}
\end{array}\right),
$$

so that the total induced dipole moment of the pair $\mathbf{p}_{1}+\mathbf{p}_{2}$ can be written as $\boldsymbol{\alpha}^{\text {es }} \cdot \mathbf{E}$, where the "electrostatic" dimer polarizability $\boldsymbol{\alpha}^{\text {es }}$ is given by the sum of the four submatrices of the inverse matrix in Eq. (10). The components of this polarizability with respect to the dimer axis system have been evaluated with the components of the dipole tensor calculated between all pairs of atoms on the two molecules and then averaged over all nine values. Relative to the noninteracting dimer, the electrostatic interaction decreases the $x x$ and $z z$ components (in the axis system of Fig. 1 and Table I) by $5 \%$ and increases the $y y$ component by $13 \%$. These changes are more extreme than those produced by the full interaction in the interacting dimer, namely decreases of $3 \%$ in the $x x$ and $z z$ components and an increase of $8 \%$ in the $y y$ component, although each approach yields a change of only $+1 \%$ in the trace of the polarizability.

Similarly, one can calculate the dipole moments induced in the dimer of molecules interacting purely electrostatically.
If the permanent moments of the noninteracting molecules are $\boldsymbol{\mu}_{1}^{0}$ and $\boldsymbol{\mu}_{2}^{0}$, which become $\boldsymbol{\mu}_{1}$ and $\boldsymbol{\mu}_{2}$ in the dimer without any external field, we have

$$
\boldsymbol{\mu}_{1}=\boldsymbol{\mu}_{1}^{0}+\boldsymbol{\alpha}_{1} \cdot \mathbf{F}_{1}^{p}, \quad \boldsymbol{\mu}_{2}=\boldsymbol{\mu}_{2}^{0}+\boldsymbol{\alpha}_{2} \cdot \mathbf{F}_{2}^{p} .
$$

Here $\mathbf{F}_{1}^{p}$ and $\mathbf{F}_{2}^{p}$ are the permanent fields in the dimer due to the dipole moments, given by $\mathbf{T} \cdot \boldsymbol{\mu}_{2}$ and $\mathbf{T} \cdot \boldsymbol{\mu}_{1}$, respectively. Substituting in Eq. (11) and solving yields

$$
\begin{aligned}
\left(\begin{array}{l}
\boldsymbol{\mu}_{1} \\
\boldsymbol{\mu}_{2}
\end{array}\right) & =\left(\begin{array}{cc}
\mathbf{1} & -\boldsymbol{\alpha}_{1} \cdot \mathbf{T} \\
-\boldsymbol{\alpha}_{2} \cdot \mathbf{T} & \mathbf{1}
\end{array}\right)^{-1}\left(\begin{array}{c}
\boldsymbol{\mu}_{1}^{0} \\
\boldsymbol{\mu}_{2}^{0}
\end{array}\right) \\
& =\left(\begin{array}{cc}
\boldsymbol{\alpha}_{1}^{-1} & -\mathbf{T} \\
-\mathbf{T} & \boldsymbol{\alpha}_{2}^{-1}
\end{array}\right)^{-1}\left(\begin{array}{cc}
\boldsymbol{\alpha}_{1}^{-1} & \mathbf{0} \\
\mathbf{0} & \boldsymbol{\alpha}_{2}^{-1}
\end{array}\right)\left(\begin{array}{l}
\boldsymbol{\mu}_{1}^{0} \\
\boldsymbol{\mu}_{2}^{0}
\end{array}\right),
\end{aligned}
$$

where $\mathbf{1}$ and $\mathbf{0}$ are the $3 \times 3$ unit and null matrices, respectively; the second expression shows that the inverse already required for the induced dipoles can be used for the permanent dipoles too. This expression can also be manipulated to yield directly the dipole moment changes $\delta \boldsymbol{\mu}_{1}$ and $\delta \boldsymbol{\mu}_{2}$ in the dimer as

$$
\left(\begin{array}{l}
\delta \boldsymbol{\mu}_{1} \\
\delta \boldsymbol{\mu}_{2}
\end{array}\right)=\left(\begin{array}{ll}
\boldsymbol{\alpha}_{1}^{-1} & -\mathbf{T} \\
-\mathbf{T} & \boldsymbol{\alpha}_{2}^{-1}
\end{array}\right)^{-1}\left(\begin{array}{cc}
\mathbf{0} & \mathbf{T} \\
\mathbf{T} & \mathbf{0}
\end{array}\right)\left(\begin{array}{l}
\boldsymbol{\mu}_{1}^{0} \\
\boldsymbol{\mu}_{2}^{0}
\end{array}\right) .
$$

This shows the changes as the self-consistent electrostatic response of the dimer to the field of the permanent dipole moments. The dipole moments are dominated by the $y$ component, which increases in magnitude by $13 \%$ with the purely electrostatic interaction, but by $19 \%$ with the full interaction in the interacting dimer, where the transfer of charge between molecules enhances the effect.

The TPEP calculations we have reported take no account of correlation, which our present techniques cannot handle. Correlation affects the polarizabilities directly and also indirectly through the calculated equilibrium geometry. For the interacting dimer held at the old equilibrium geometry, taking account of correlation at MP2 level increases the diagonal components of the total polarizability by about $15 \%$ each. In the equilibrium geometry calculated taking account of correlation at the MP2 level (where the major change is a shortening in the $\mathrm{O}-\mathrm{O}$ distance along the $y$ axis), the $x x$ and $z z$ components of the total dimer polarizability increase by $18 \%$ and the $y y$ component by $21 \%$. In each case, the polarizabilities for the interacting dimer change relative to those for the noninteracting dimer by only about two-thirds as much as the electrostatic model predicts (using the MP2 corrected polarizabilities for the molecules in the noninteracting dimer), which is essentially the same proportion as in the absence of correlation.

\section{DISCUSSION AND CONCLUSIONS}

We have presented extensive results from calculations of the static distributed polarizability in the calculated equilibrium structure of the water dimer, and, for comparison, in the isolated water molecule and in a dimer of noninteracting water molecules. The results satisfy two tests, among others. First, the molecular polarizability reconstructed from the distributed components is in excellent agreement with the result from CPHF calculations, and secondly, the total charge re- 
mains unchanged after redistribution within the dimer. Although our distributed polarizability calculations do not take account of correlation, our MP2 calculations indicate that the main effect of correlation is to increase the total polarizability in magnitude fairly uniformly in the dimer $x z$ plane, with a slight enhancement along the $y$ axis, which is the hydrogen bond direction where the geometry changes most. This suggests that our TPEP calculations ignoring correlation should give a good qualitative picture of the distributed polarizability.

We find that hydrogen bonding has the effect of decreasing the overall polarizability, owing to reorganization of charge density along the hydrogen bond. The effect is most significant along the direction of the bonding, and is larger for the water molecule in which the oxygen atom is involved in the hydrogen bonding (Molecule 1). This decrease in polarizability occurs at the same time as the well-known increase in dipole moment. Placing the dimer in a uniform external field shows that charge flows predominantly along the hydrogen bond in the direction of the field, thereby inducing a difference in net charge between molecules. Comparing results for the interacting dimer with those for the noninteracting dimer supplemented by purely electrostatic interactions shows that the additional interactions oppose the effects of the electrostatic interactions in the polarizability but enhance them in the dipole moment. This suggests that the permanent charge-transfer between molecules in the dimer, which certainly enhances the dipole moment, may have the opposite effect on the polarizability.

These results should be understood in the context of the method we use. When an electric field is applied to a molecule, two things happen to the atomic properties, in the AIM picture. One is that properties within the basin such as charge and dipole moment change, and the other is that the atomic basin itself changes. These give rise to what have been called basin and surface contributions to the atom polarizabilities $^{3}$ whereas what we calculate corresponds to the basin contributions alone. ${ }^{2}$ Calculations indicate that surface contributions are often small, but not negligible, especially if the atoms have very different electronegativities, ${ }^{40}$ and more recent work has derived and implemented a method valuable for calculating surface terms accurately using analytical rather than numerical differentiation. ${ }^{41,42}$ However, the molecular properties obtained from the basin contributions alone are the same as those obtained from basin and surface contributions together, essentially because the net contribution over the surface shared by two atoms is zero. This means that basin contributions by themselves do afford a legitimate method of partitioning the molecular response among what might be termed "frozen atoms," and it is in that context that we have discussed them.

As noted in the Introduction, calculation of distributed polarizability is desirable for practical applications. For example, Karlström has calculated distributed polarizabilities using the uncoupled Hartree-Fock method ${ }^{43}$ and used them to devise the polarizable water potential non-emperical molecular orbital (NEMO). ${ }^{44}$ Calculation of distributed polarizability is also desirable for conceptual understanding of how the electron distribution responds to the external influence of electric fields, affording insights that go beyond those available from analysis of the ground-state electron distribution. The calculation of the atomic and molecular polarizability of the dimer (and by implication of other sets of molecules) also proves to be a powerful tool for providing insight into the effect of interaction on the electron density and its response to electric fields. In particular, the separate molecular polarizabilities in the dimer can be calculated from the distributed components of the molecular polarizability of the dimer, which is not possible using standard $a b$ initio packages.

Various extensions of the present work can be envisaged. We have already calculated nonlinear response in the form of distributed hyperpolarizabilities for urea, which prove to have a significant effect on the calculated nonlinear response of the crystal. ${ }^{45}$ In this respect there is a need to extend the method to frequency-dependent response. It is also desirable to make the method more efficient, perhaps by using direct methods to compute integrals as required, so that high-level calculations on larger molecules and clusters become less demanding of computer resources.

\section{ACKNOWLEDGMENTS}

We thank G. Jansen and C. Hättig for useful comments. M.i.h.P. acknowledges support from the EU TMR DELOS Network Program under Contract Number FMRX-CT960047.

\section{APPENDIX: DERIVATION OF POLARIZABILITY IN MOLECULAR ORBITAL (MO) DESCRIPTION}

Suppose a molecule is perturbed by a static electric field. The energy of the molecule can then be expanded according to

$E=E^{(0)}-\mu_{\alpha}^{(0)} F_{\alpha}-\frac{1}{2} \alpha_{\alpha \beta} F_{\alpha} F_{\beta}-\frac{1}{6} \beta_{\alpha \beta \gamma} F_{\alpha} F_{\beta} F_{\gamma}-\cdots$,

where subscripts $\alpha$ and $\beta$ denote Cartesian components. $E^{(0)}$ is the unperturbed energy, $F_{\alpha}$ is the component of the field in the $\alpha$ direction, $\mu_{\alpha}^{(0)}$ is the component of the permanent dipole moment of the molecule in the $\alpha$ direction, and $\alpha_{\alpha \beta}$ and $\beta_{\alpha \beta \gamma}$ are static polarizability and first hyperpolarizability tensor components. The polarizability is related to the second-order energy by

$$
E^{(2)}=-\frac{1}{2} \alpha_{\alpha \beta} F_{\alpha} F_{\beta}
$$

and can be calculated exactly using the sum over states (SOS) expression. ${ }^{19}$ The SOS expression for the secondorder energy can be found using standard RayleighSchrödinger perturbation theory as

$$
E^{(2)}=\sum_{n \neq 0} \frac{\left\langle 0\left|H^{\prime}\right| n\right\rangle\left\langle n\left|H^{\prime}\right| 0\right\rangle}{E_{n}-E_{0}} .
$$

This is not practical if one wishes to calculate reliable polarizabilities, although a promising approximate SOS approach in organic molecules was presented recently. ${ }^{46}$

The SOS expression can be converted into a CPHF expression, as shown by Silvi and Fourati. ${ }^{7}$ The SOS expression is defined in exact wave functions, whereas the CPHF expression is defined in terms of molecular orbitals (MOs). 
This gives a practical manner of calculation since the MOs are easily obtained from a standard $a b$ initio package. With increasing the level of sophistication of the basis set the result will become more exact.

Alternatively, the polarizability can be obtained through partial derivatives of the energy expansion Eq. (A1)

$$
-\alpha_{\alpha \beta}=E^{\alpha \beta}=2 \sum_{s t}^{\text {basis }} D_{s t}^{\beta} h_{s t}^{\alpha},
$$

where $E^{\alpha \beta}, D^{\beta}$, and $h^{\alpha}$ are derivatives of the energy, density matrix and Hamiltonian, respectively. The summation is over the basis functions $s t$. Equation (A4) defines the polarizability in tensor trace formalism. ${ }^{8}$ The density matrix is given by

$$
D_{s t}^{\beta}=\sum_{k}^{\text {occ }}\left(C_{s k}^{\beta^{*}} C_{t k}+C_{s k}^{*} C_{t k}^{\beta}\right),
$$

with $C_{s k}^{\beta^{*}}$ a perturbed MO coefficient and $C_{t k}$ an unperturbed MO coefficient. The summation is over occupied MOs. Substitution into Eq. (A4) leads to:

$$
E^{\alpha \beta}=2 \sum_{s t}^{\text {basis occ }} \sum_{k}\left(C_{s k}^{\beta^{*}} C_{t k} h_{s t}^{\alpha}+C_{s k}^{*} C_{t k}^{\beta} h_{s t}^{\alpha}\right) .
$$

The procedure to convert this equation into a form that can be readily calculated by an ab initio program does not appear to have been reported in full. Hence we give the steps necessary to transform Eq. (A6) into a molecular orbital description that can be readily calculated.

It can be shown that both terms in Eq. (A6) give equal contributions, which yields

$$
E^{\alpha \beta}=4 \sum_{s t}^{\text {basis occ }} \sum_{k} C_{s k}^{\beta^{*}} C_{t k} h_{s t}^{\alpha} .
$$

Now this summation over basis functions can be changed into a summation over MOs, by first expanding the Hamiltonian in terms of basis functions

$$
\begin{aligned}
E^{\alpha \beta} & =4 \sum_{s t}^{\text {basis }} \sum_{k}^{\text {occ }} C_{s k}^{\beta^{*}} C_{t k} h_{s t}^{\alpha} \\
& =4 \sum_{s t}^{\text {basis occ }} \sum_{k} C_{s k}^{\beta^{*}} C_{t k} \int \chi_{s}^{*} \hat{H}^{\alpha} \chi_{t} d \tau,
\end{aligned}
$$

and, second, using the definition of a MO

$$
\phi_{k}=\sum_{s}^{\text {basis }} \chi_{s} C_{s k}
$$

Equation (A8) then becomes

$$
E^{\alpha \beta}=4 \sum_{k}^{\text {occ }}\left\langle\phi_{k}^{\beta}\left|\hat{H}^{\alpha}\right| \phi_{k}\right\rangle .
$$

In static perturbation ${ }^{9,10,47}$ a perturbed orbital $\phi_{k}^{\beta}$ is expanded in the basis of the unperturbed orbitals as follows: ${ }^{7}$

$$
\left\langle\phi_{k}^{\beta}\right|=\sum_{\sigma}^{\text {vir }} U_{k \sigma}^{\beta^{*}}\left\langle\phi_{\sigma}\right|,
$$

where the summation is over virtual MOs. The transformation matrix $U_{k \sigma}^{\beta^{*}}$ can be found by solving the coupled perturbed Hartree-Fock equations which are of the general form $^{7}$

$$
\sum_{l}^{\text {occ }} \sum_{\tau}^{\text {vir }} G_{k \sigma, l \tau} U_{l \tau}^{\beta}=h_{k \sigma}^{\beta},
$$

which can be inverted to yield

$$
U_{k \sigma}^{\beta}=\sum_{l}^{\text {occ }} \sum_{\tau}^{\text {vir }}\left(\mathbf{G}^{-1}\right)_{k \sigma, l \tau} h_{l \tau}^{\beta} .
$$

The Hamiltonian is Hermitian, and we, therefore, obtain

$$
U_{k \sigma}^{\beta^{*}}=\sum_{l}^{\text {occ }} \sum_{\tau}^{\text {vir }}\left(\mathbf{G}^{-1}\right)_{k \sigma, l \tau} h_{\tau l}^{\beta} .
$$

Substitution of Eqs. (A14) and (A11) into Eq. (A10) yields

$$
-\alpha_{\alpha \beta}=E^{\alpha \beta}=4 \sum_{k l}^{\text {occ }} \sum_{\sigma \tau}^{\text {vir }}\left\langle\sigma\left|\hat{H}^{\alpha}\right| k\right\rangle\left(\mathbf{G}^{-1}\right)_{k \sigma, l \tau}\left\langle\tau\left|\hat{H}^{\beta}\right| l\right\rangle,
$$

where $\left\langle\sigma\left|\hat{H}^{\alpha}\right| k\right\rangle=\left\langle k\left|\hat{H}^{\alpha}\right| \sigma\right\rangle$ because of the Hermiticity of the Hamiltonian. Finally we arrive at the following result, which is similar to that in Ref. 2

$$
-\alpha_{\alpha \beta}=4 \sum_{k \sigma} \sum_{l \tau}\left\langle k\left|\hat{H}^{\alpha}\right| \sigma\right\rangle\left(\mathbf{G}^{-1}\right)_{k \sigma, l \tau}\left\langle l\left|\hat{H}^{\beta}\right| \tau\right\rangle .
$$

Here we have used the same convention for summation over all molecular orbitals. Note that in the derivation of higherorder properties such as the first hyperpolarizability it becomes more convenient to use the summation convention of Eq. (A15). Equation (A16) has also been derived by Thomsen and Swanstrøm. ${ }^{10}$ Converting to Stone's multipole moments description used to calculate distributed properties is then a straightforward operation by replacing the Hamiltonian operator by a multipole moment operator in an atomic basin. ${ }^{1}$

${ }^{1}$ A. J. Stone, Mol. Phys. 56, 1065 (1985).

${ }^{2}$ J. G. Ángyán, G. Jansen, M. Loos, C. Hättig, and B. A. Heß, Chem. Phys. Lett. 219, 267 (1994).

${ }^{3}$ R. W. F. Bader, Atoms in Molecules-A Quantum Theory (Oxford University Press, Oxford, 1990).

${ }^{4}$ P. L. A. Popelier, Atoms in Molecules-An Introduction (Pearson Education, Harlow, 2000).

${ }^{5}$ G. J. B. Hurst, M. Dupuis, and E. Clementi, J. Chem. Phys. 89, 385 (1988).

${ }^{6}$ S. P. Karna and M. Dupuis, J. Comput. Chem. 12, 487 (1990).

${ }^{7}$ B. Silvi and N. Fourati, Mol. Phys. 52, 415 (1984).

${ }^{8} \mathrm{R}$. McWeeny, Methods of Molecular Quantum Mechanics (Academic, New York, 1989).

${ }^{9}$ J. Gerratt and I. M. Mills, J. Chem. Phys. 49, 1719 (1968).

${ }^{10}$ K. Thomsen and P. Swanstrøm, Mol. Phys. 26, 735 (1973).

${ }^{11}$ C. E. Dykstra and P. G. Jasien, Chem. Phys. Lett. 109, 388 (1984).

${ }^{12}$ G. Jansen, C. Hättig, B. A. Heß, and J. G. Ángyán, Mol. Phys. 88, 69 (1996).

${ }^{13}$ C. Hättig, G. Jansen, B. A. Heß, and J. G. Ángyán, Mol. Phys. 91, 145 (1997).

${ }^{14}$ A. J. Stone, C. Hättig, G. Jansen, and J. G. Ángyán, Mol. Phys. 89, 595 (1996).

${ }^{15}$ C. Chipot, J. G. Ángyán, and C. Millot, Mol. Phys. 94, 881 (1998).

${ }^{16}$ C. Hättig, G. Jansen, B. A. Heß, and J. G. Ángyán, Can. J. Chem. 74, 976 (1996). 
${ }^{17}$ J.-C. Soetens, G. Jansen, and C. Millot, Mol. Phys. 96, 1003 (1999).

${ }^{18}$ E. R. Batista, S. S. Xantheas, and H. Jónsson, J. Chem. Phys. 111, 6011 (1999).

${ }^{19}$ R. Specchio, A. Famulari, M. Sironi, and M. Raimondi, J. Chem. Phys. 111, 6204 (1999).

${ }^{20}$ M. Masella and J.-P. Flament, J. Chem. Phys. 107, 9105 (1997).

${ }^{21}$ A. Milet, R. Moszynski, P. E. S. Wormer, and A. van der Avoird, J. Phys. Chem. A 103, 6811 (1999).

${ }^{22}$ K. Hermansson and M. Alfredsson, J. Chem. Phys. 111, 1993 (1999).

${ }^{23}$ A. Szabo and N. S. Ostlund, Modern Quantum Chemistry (McGraw-Hill, London, 1989).

${ }^{24}$ M. J. Frisch, G. W. Trucks, H. B. Schlegel et al. GAUSSIAN 94 (Revision A.1), Gaussian Inc., Pittsburgh, PA, 1995.

${ }^{25}$ MORPHY01, a program written by P. L. A. Popelier with a contribution from R. G. A. Bone, D. Kosov and M. in het Panhuis, UMIST, Manchester, U.K. (2000).

${ }^{26}$ P. L. A. Popelier, Mol. Phys. 87, 1169 (1996).

${ }^{27}$ P. L. A. Popelier, Comput. Phys. Commun. 108, 180 (1998).

${ }^{28}$ G. Jansen, C. Hättig, and J. G. Ángyán, CAUCHY-A TDHF program for distributed polarizabilities (1994).

${ }^{29}$ A. J. Sadlej, Collect. Czech. Chem. Commun. 53, 1995 (1988).

${ }^{30}$ C. R. Le Sueur and A. J. Stone, Mol. Phys. 78, 1267 (1993).

${ }^{31}$ P. M. W. Gill, J. Phys. Chem. 100, 15421 (1996).

${ }^{32}$ C. Millot and A. J. Stone, Mol. Phys. 77, 439 (1992).
${ }^{33}$ E. M. Mas, R. Bukowski, K. Szalewicz, G. C. Groenenboom, P. E. S. Wormer, and A. van der Avoird, J. Chem. Phys. 113, 6687 (2000).

${ }^{34}$ P. L. Silvestrelli and M. Parrinello, J. Chem. Phys. 111, 3572 (1999).

${ }^{35}$ E. R. Batista, S. S. Xantheas, and H. Jónsson, J. Chem. Phys. 112, 3285 (2000).

${ }^{36}$ W. G. J. Hol, P. T. van Duijnen, and H. J. C. Berendsen, Nature (London) 273, 443 (1978).

${ }^{37}$ E. J. Milner-White, Protein Sci. 6, 2477 (1997).

${ }^{38}$ L. Silberstein, Philos. Mag. 33, 521 (1917).

${ }^{39}$ A. D. Buckingham, Trans. Faraday Soc. 52, 747 (1956).

${ }^{40}$ K. E. Laidig and R. F. W. Bader, J. Chem. Phys. 93, 7213 (1990).

${ }^{41}$ J. Cioslowski, Mol. Phys. 88, 621 (1996).

${ }^{42}$ J. Cioslowski and B. B. Stefanov, J. Chem. Phys. 105, 8741 (1996)

${ }^{43}$ G. Karlström, Theor. Chim. Acta 60, 535 (1982).

${ }^{44}$ M. A. Carignano, G. Karlström, and P. Linse, J. Phys. Chem. B 101, 1142 (1997).

${ }^{45}$ H. Reis, M. G. Papadopoulos, C. Hättig, J. Ángyán, and R. W. Munn, J. Chem. Phys. 112, 6161 (2000).

${ }^{46}$ G. P. Das and D. S. Dudis, Chem. Phys. Lett. 312, 57 (1999).

${ }^{47}$ R. M. Stevens, R. M. Pitzer, and W. N. Lipscomb, J. Chem. Phys. 38, 550 (1963).

${ }^{48}$ C. G. Gray and K. E. Gubbins, Theory of Molecular Fluids, Vol. 1 (Clarendon, London, 1984). 